\title{
The effect of altered head and tongue posture on upper airway volume based on a validated upper airway analysis- An MRI pilot study
}

\section{Running title: Head and tongue posture and UA Volume}

Sirwan Fernandez Gurani, DDS, PhD fellow, ${ }^{\mathrm{a}}$

Paolo Maria Cattaneo, Associate Professor, ${ }^{b}$

Søren Rafael Rafaelsen, Professor, DMSc, MD, ${ }^{c}$

Malene Roland Pedersen, PhD, Radiographer, ${ }^{\mathrm{c}}$

Jens Jørgen Thorn, DDS, PhD, Senior Consultant, ${ }^{a}$

Else Marie Pinholt, Professor, Dr. odont, M.Sci, a,d

${ }^{a}$ Department of Oral \& Maxillofacial Surgery, Hospital of South West Denmark, Finsensgade 35, 6700, Esbjerg, Denmark \& University of Southern Denmark, Faculty of Health Sciences, Institute of Regional Health Services Sciences, Denmark.

Email: sirwan.fernandez.gurani@,rsyd.dk.

${ }^{\mathrm{b}}$ Section of Orthodontics, Department of Dentistry and Oral Health, Faculty of Health Science, Aarhus University, Vennelyst Boulevard, 8000 Aarhus C, Denmark.

${ }^{\mathrm{c}}$ Department of Radiology, Vejle Hospital, Beriderbakken 4, 7100 Vejle, Denmark \& Institute of Regional Health Research, University of Southern Denmark, Odense, Denmark.

${ }^{\mathrm{d}}$ Department of Oral \& Maxillofacial Surgery, Hospital of South West Denmark, Finsensgade 35, 6700 Esbjerg, Denmark \& University of Southern Denmark, Faculty of Health Sciences, Institute of Regional Health Services Sciences, Denmark.

\section{Corresponding author:}

Sirwan Fernandez Gurani

Department of Oral \& Maxillofacial Surgery

This is the author manuscript accepted for publication and has undergone full peer review but has not been through the copyediting, typesetting, pagination and proofreading process, which may lead to differences between this version and the Version of Record. Please cite this article as doi: $\underline{10.1111 / \text { OCR.12348 }}$

This article is protected by copyright. All rights reserved 
Hospital of South West Denmark

Finsensgade 35,

6700, Esbjerg, Denmark

Phone work: +4579182145

Phone private: +4560656112

E-mail: sirwan.fernandez.gurani@,rsyd.dk

\section{Declaration of interest: None}

Funding: This work is part of a $\mathrm{PhD}$ project, which was supported by the Region of Southern Denmark (grant number J.nr. 15/51299, 2015)

Permission from the Ethical Committees: The study was approved by the Regional Committees on Health Research Ethics for Southern Denmark (Project-ID S-20150076) and conducted in accordance to the Helsinki Declaration comprising healthy volunteer colleagues from our research team and from the Department of Oral and Maxillofacial Surgery, Hospital of South West Denmark, Esbjerg, Denmark.

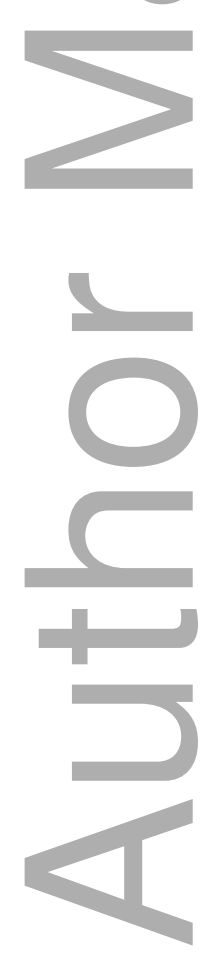




\begin{abstract}
OBJECTIVE To determine the influence of altered head or tongue posture on the upper airway (UA) volumes using MRI imaging based on a new objective and validated UA evaluation protocol. SETTING AND SAMPLE POPULATION One supine CBCT and five sagittal MRI scans were obtained from ten subjects in different head and tongue positions: (1) supine neutral head position (NHP) with the tongue in a natural resting position with tip of the tongue in contact with the lingual aspect of the lower incisors (TRP); (2) Head extension with TRP; (3) Head flexion with TRP; (4) NHP with the tip of the tongue in contact with the posterior edge of the hard palate (THP); and (5) NHP with the tip of the tongue in contact with the floor of the mouth in contact with Caruncula sublingualis.
\end{abstract}

MATERIAL AND METHODS Based on a validated CBCT UA analysis, the retropalatal-, oropharyngeal- and the corresponding total volumes, were measured from each MRI scan. Wilcoxon sign-rank test was applied to determine the statistically significant difference in mean volume between the baseline head and tongue posture (NHP with TRP) and the other postures. RESULTS Five females and five males with a mean age of 46,5 \pm 13.7 years volunteered for this pilot study. The UA volumes, particularly the oropharyngeal volume, increased significantly with head extension and NHP with THP, and decreased significantly with head flexion.

CONCLUSION Altered head and tongue posture proved to affect the UA volumes thus representing confounding variables during three-dimensional radiographic image acquisition.

KEYWORDS: Magnetic Resonance Imaging, Cone-Beam CT, Cephalometry, Posture, Pharynx.

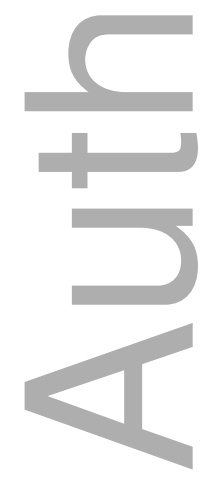


DR SIRWAN FERNANDEZ GURANI (Orcid ID : 0000-0003-3121-1620)

Article type : Original Article

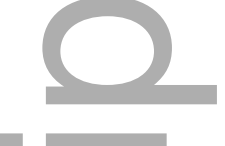

The effect of altered head and tongue posture on upper airway volume based on a validated upper airway analysis- An MRI pilot study
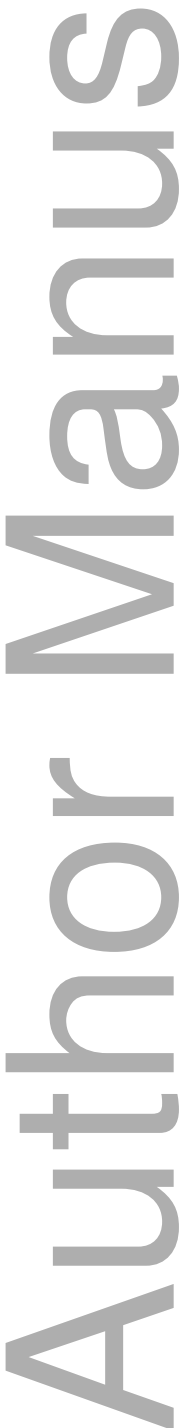

This article is protected by copyright. All rights reserved 


\begin{abstract}
OBJECTIVE To determine the influence of altered head or tongue posture on the upper airway (UA) volumes using MRI imaging based on a new objective and validated UA evaluation protocol.

SETTING AND SAMPLE POPULATION One supine CBCT and five sagittal MRI scans were obtained from ten subjects in different head and tongue positions: (1) supine neutral head position (NHP) with the tongue in a natural resting position with tip of the tongue in contact with the lingual aspect of the lower incisors (TRP); (2) Head extension with TRP; (3) Head flexion with TRP; (4) NHP with the tip of the tongue in contact with the posterior edge of the hard palate (THP); and (5) NHP with the tip of the tongue in contact with the floor of the mouth in contact with Caruncula sublingualis.
\end{abstract}

MATERIAL AND METHODS Based on a validated CBCT UA analysis, the retropalatal-, oropharyngeal- and the corresponding total volumes, were measured from each MRI scan. Wilcoxon sign-rank test was applied to determine the statistically significant difference in mean volume between the baseline head and tongue posture (NHP with TRP) and the other postures.

RESULTS Five females and five males with a mean age of 46,5 \pm 13.7 years volunteered for this pilot study. The UA volumes, particularly the oropharyngeal volume, increased significantly with head extension and NHP with THP, and decreased significantly with head flexion.

CONCLUSION Altered head and tongue posture proved to affect the UA volumes thus representing confounding variables during three-dimensional radiographic image acquisition.

KEYWORDS: Magnetic Resonance Imaging, Cone-Beam CT, Cephalometry, Posture, Pharynx.

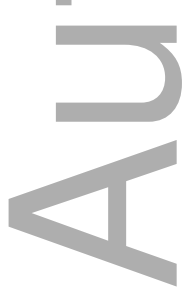




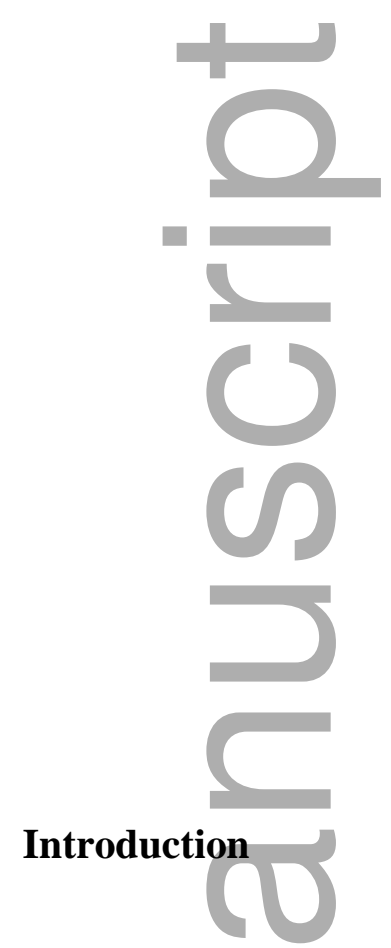

Cone beam computed tomography (CBCT) has emerged as a common imaging modality for 3D cephalometric analysis and UA assessment due to its high-resolution visualization of bony structures, teeth and air cavities. ${ }^{1,2}$

The use of CBCT is associated with low emission of radiation dosage nevertheless recent literature has an increased focus on ionizing radiation from dental CBCT as a carcinogenesis risk in children, and has recommended increased awareness and the principles of good radiation practice. ${ }^{3}$

MRI obtains 3D images of the surrounding soft-tissue structures of the UA with excellent contrast due to high soft-tissue differentiation and contrast resolution though with lower inter-slice resolution. ${ }^{4,5}$ Hence MRI has been applied in several UA studies to assess the UA dimensions and morphology in subjects with obstructive sleep apnea (OSA). ${ }^{6,7}$

Numerous factors including head, tongue and body posture, respiratory phase and deglutition may influence UA dimensions and morphology during image acquisition. ${ }^{2,8-11}$ 
Natural head position (NHP) is convenient as a standardized standing/seating image acquisition posture for cephalometric analysis and UA assessment ${ }^{2,12}$ which has been shown to be the most accurate and reproducible head position. ${ }^{13}$

Head extension is defined as an increase of the cranio-cervical angle (NSL/OTP) whereas head flexion is a reduction of the cranio-cervical angle. ${ }^{14}$

UA dimensions and morphology seem to be affected by altered head posture when evaluated on two-dimensional (2D) imaging. ${ }^{15,16}$ However this association should be evaluated using 3D imaging techniques since 2D images are associated with several drawbacks including distortion, magnification and superimposition of bilateral craniofacial structures and lack of information regarding UA volume and cross-sectional area (CSA). 1,2,17-19

A systematic literature review ${ }^{20}$ revealed limited studies regarding the impact of altered head posture and none on altered tongue posture on the UA dimensions and morphology during 3D imaging. Non-comparable studies with heterogeneous methodology and UA estimations based on undefined tongue postures during image acquisition were the result of this literature review. ${ }^{11,21,22}$

The possible effect of tongue posture on the UA measures has been stressed in several studies. ${ }^{2,23-25}$ Moreover the genioglossus muscle activity and motion was associated with altered head and body posture. ${ }^{10,26}$ This was described as the main UA dilator muscle, thus playing an important role in the maintenance of the UA patency by increasing the anteroposterior dimension at the level of the oropharynx. ${ }^{27-29}$

The impact of postural changes on the UA has been studied using upright CBCT and supine CT scanners. ${ }^{30}$ This study demonstrated a significantly larger oropharyngeal dimension at the level of the soft palate in upright body position than in supine position. In addition, the soft palate, epiglottis and hyoid bone were also displaced due to the gravitational changes.

Altered head or tongue posture may thus represent confounding factors during 3D image acquisition. Comparison and reliability of UA measurements and outcomes may therefore be questioned and future investigation has been recommended. 1,2,18,20 
A new objective CBCT UA protocol was recently developed and validated with the purpose of measuring the UA volume, CSA and linear measurements in patients following orthognathic surgery. ${ }^{31}$ The method was accordingly applied to a retrospective sample to evaluate the effect of bimaxillary surgery on the UA volumes, CSA and positional changes of epiglottis and hyoid bone. ${ }^{32}$

In order to obtain reliable and comparable MRI and CBCT images with valid outcomes, the association between altered head or tongue posture and UA volumes, ought to be clarified based on an objective and verified UA analysis.

The aim of this pilot study was therefore to determine the impact of altered head or tongue posture on the UA volumes obtained from MRI scans based on a standardized and validated UA analysis. ${ }^{31}$

The authors hypothesized that altered head or tongue posture has an impact on the UA volumes.
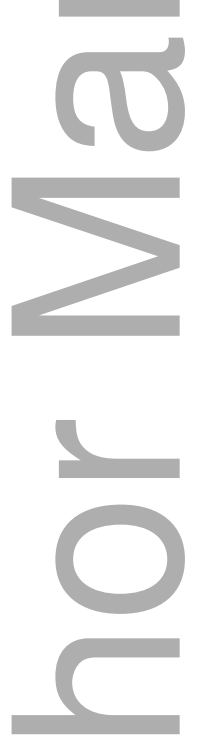

\section{Material and Methods}

Study sample

This prospective clinical pilot study was approved by the Regional Committees on Health Research Ethics for Southern Denmark (Project-ID S-20150076), comprising healthy volunteer colleagues from our research team and from the Department of Oral and Maxillofacial Surgery, Hospital of South West Denmark, Esbjerg, Denmark.

Written informed consent was obtained from the study subjects. Inclusion criteria required the participants to be healthy and 18 years or older, neither be pregnant nor contain any 
ferrous or iron-containing metal implants not considered MRI-safe. The predictor variables were altered head- and tongue postures and the outcome variable was the UA volume. All data for this study were anonymized.

\section{MRI image acquisition}

The MRI scans were acquired at the Department of Radiology, Vejle Hospital - Part of Lillebaelt Hospital, Vejle, University Hospital of Southern Denmark, with an Ingenia 3.0 Tesla (T) MR system (Philips Healthcare, Best, The Netherlands). T2-weighted images were acquired in sagittal plane with a field of view (FOV) of $23 \mathrm{~cm}$, comprising 39 slices with 3,6 $\mathrm{mm}$ increment and $3.0 \mathrm{~mm}$ of thickness. The participants were scanned with a neurovascular head coil, which permitted the change of head positions, and the scanning time was approximately 4 minutes for each head- and tongue position.

As presented in Figure 1, a total of five MRI scans were taken for each subject, with the subjects lying in a supine position. During each scan, the subjects were asked to have the head and tongue in five well-defined positions as in the following: (1) NHP with the tongue in a natural resting position (TRP - i.e. with tip of the tongue in contact with the lingual aspect of the lower incisors); (2) Head extension with TRP; (3) Head flexion with TRP; (4) NHP with the tip of the tongue in contact with the posterior edge of the hard palate (THP) and (5) NHP with the tip of the tongue in contact with the floor of the mouth in contact with Caruncula sublingualis (TFM).

The first position (i.e. NHP with TRP) represented the baseline to which the other head and tongue positions were compared.

During the head extension and head flexion, the participants were instructed to tilt the head backwards and forwards at a maximum and to keep the teeth in intercuspation, avoid deglutition and head or body movements and maintain quiet nasal breathing during image acquisitions

Supine CBCT image acquisition

A supine CBCT scan was presented from each participant, which was acquired as part of another study to compare CBCT with MRI UA parameters

The CBCT scans were acquired at the Section of Oral Radiology, Department of Dentistry and Oral Health, Aarhus University, Aarhus, Denmark, with a NewTom ${ }^{\mathrm{TM}} 5 \mathrm{G}(\mathrm{QR}$ Srl, Verona, Italy), FOV of $18.3 \mathrm{~cm}$, X-ray tube current 3-7 mA, KVP $110 \mathrm{kV}, 538$ slices of 
$0.300 \mathrm{~mm}$ increment and thickness $0.300 \mathrm{~mm}$, thus producing isotropic voxels. Scanning time was approximately $18 \mathrm{sec}$, with actual radiation time of $3.6 \mathrm{sec}$.

The participants were scanned one time in a supine position; with NHP with TRP as shown in the CBCT scan Figure 1. During image acquisition, the participants were instructed to keep the teeth in intercuspation, avoid deglutition and head or body movements and maintain quiet nasal breathing.

MRI- and CBCT raw-data were transferred from the scanners via the DICOM format and imported into Mimics Research 19.0 (Materialise, Leuven, Belgium) for UA analysis.

UA analysis

The first author performed the UA analysis on each MRI scan applying a validated UA analysis. ${ }^{31}$ The CBCT scans were applied in order to increase the accuracy of identifying and selecting the corresponding landmarks on the MRI-scans, and thus the CBCT scans served as a complementary quality assurance in this study.

As presented in Figure 2, this analysis involved identifying several stable anatomical landmarks followed by generation of a series of reference planes in order to delimit the superior- and inferior border of the UA and subdivide the UA into a retropalatal-, an oropharyngeal and a total volume compartment which comprised the outcome variables of this pilot study.

\section{Statistical analysis}

Descriptive statistics included the participants' mean age \pm SD (range) at time of image acquisition and the mean volume obtained for each UA compartment at each specified headand tongue positions.

The volume in each UA compartment, the retropalatal-, the oropharyngeal- and the total volume, were considered the baseline size with reference to the posture 'NHP with TRP'. The Stata/IC 15.1 for Mac StataCorp LLC was used for the statistical analysis, which comprised a non-parametric statistical test due to a small sample size.

With a significance threshold set at $\mathrm{P} \leq .05$, the Wilcoxon signed-rank test was applied to determine whether the mean volume differences from each UA volume compartment were statistically significant different when the subjects changed from baseline head- and tongue posture to the other specified head- and tongue postures.

\section{Results}

This article is protected by copyright. All rights reserved 
Ten subjects comprising five females and five males with a mean age of $46,5 \pm 13.7$ years (range 32 to 66 years) and a body mass index $(\mathrm{BMI})<30\left(\mathrm{~kg} / \mathrm{m}^{2}\right)$ volunteered for this study. The descriptive statistics are summarized in Table I.

The effect of altered head- and tongue posture on the UA volume compartments is summarized in Table II. With the tongue in resting position the outcome variables increased significantly, Retropalatal volume $\mathrm{p}=.005$, Oropharyngeal volume $\mathrm{p}=.03$ and Total volume $\mathrm{p}=.02$, with head extension and decreased significantly, Retropalatal volume $\mathrm{p}=.006$, Oropharyngeal volume $\mathrm{p}=.009$ and Total volume $\mathrm{p}=.005$, with head flexion compared to UA volume at baseline head- and tongue posture.

With the head in NHP, the UA Total volume increased significantly, Total volume $\mathrm{p}=.03$, when the tongue was altered from a resting position (NHP with TRP) to the tip of the tongue in contact with the posterior edge of the hard palate. However no statistically significant volume differences were detected in any UA volume compartments when the tip of the tongue was moved from a resting position to contact with the floor of the mouth.
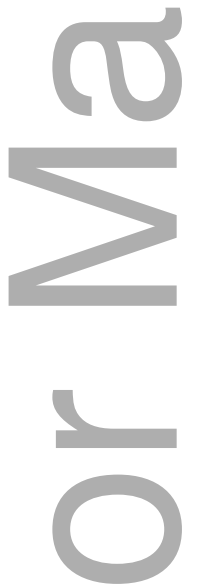

\section{Discussion}

This pilot study evaluated and compared UA volume with respect to different head and tongue postures using the MRI technique. Head extension resulted in an increase while head flexion caused a decrease of the retropalatal, oropharyngeal and hence the UA total volume when compared to NHP with the tongue in a resting position. Furthermore the UA total volume compartment increased significantly when the tip of the tongue was moved to the posterior edge of the hard palate compared to resting tongue position in NHP. Thus, our alternative hypothesis was accepted. 
Based on a validated and objective 3D UA evaluation protocol, the effect of altered head or tongue posture on the UA volume has not been thoroughly investigated although previous studies have highlighted this association $21,25,33,34$

In order to perform comparable UA studies with reliable outcomes, head posture or the cranio-cervical inclination should be in a standardized posture during image acquisition, which has been highlighted as a confounding factor in the literature ${ }^{1,18,20,31,35}$ thus calling for this study.

The genioglossus muscle electromyographic (EMG) activity has been associated with altered head rotation and body position. ${ }^{10}$ In addition, the association between head posture and the respiratory-related effect of the tongue has been demonstrated using MRI with a modified complementary spatial modulation of magnetization (CSPAMM) sequence. ${ }^{26}$ Cai et al. evaluated the effect of different head and jaw positions on the genioglossus displacement during quiet respiration. The study showed a significantly anterior displacement of the tongue during neutral position compared to extension and rotation of the head, thus indicating less required active dilation to maintain the UA patency during head extension and rotation. This stresses the importance of a standardized head posture during image acquisition.

BMI may present another potential confounder of the UA assessment however our study subjects had normal BMI.

In our study, the oropharyngeal volume compartment was more prone to the altered head and tongue posture than the retropalatal volume compartment as indicated by larger volume changes in the oropharyngeal region. This implies that associated muscle and soft tissue structures of the UA oropharyngeal region are more prone to altered head and tongue position which is in accordance with Cai et al ${ }^{26}$ that demonstrated a greater movement in the oropharyngeal tongue section compared to the UA caudal section.

Different protocols for tongue positioning during 3D image acquisition have been presented in the literature when the UA has been assessed following orthognathic surgery. ${ }^{36-38}$ These protocols include 'placing the tongue on the roof of the mouth" 36 , "placing the tongue in a relaxed position' ${ }^{37,39}$ and 'placing the tongue against the incisor teeth' ${ }^{38,40}$ which may 
complicate comparison between different studies and present bias in UA evaluation and apparently an obstacle for standardization.

During the altered head posture, the subjects performed the maximum feasible head flexion and head extension within the space of the head coil. This amount of head malposition is probably not likely to happen clinically however a standardized threshold was required although the anatomical cranio-cervical flexibility of each subject might be different.

We constructed a customised head wedge with 15 degree inclination in order to measure the head posture with 15 degrees of head extension and head flexion however the wedge was not feasible with the MRI head coil.

Nevertheless the study showed a significant volume change due to alteration of head and tongue posture and thus highlights the important message of a standardized head and tongue posture during image acquisition.

The impacts of altered head and tongue posture during image acquisition on the reliability of UA outcomes are significant which are emphasized in previous studies ${ }^{31,32}$ where a quality assessment was performed in order to exclude CBCT scans with significant malpositioned head posture (head rotation, head flexion or head extension).

The MRI or CBCT scans should be evaluated in all three planes in order to evaluate the head posture while a malpositioned tongue is easily recognised on the sagittal plane.

Therefore you cannot rely on the obtained UA data if the images before and after surgery are not standardized with regard to head and tongue posture. In addition a standardized UA analysis in different studies is essential.

Although the present study was a pilot study with limited sample size using the non-ionizing 3 Tesla MRI technique, the outcomes are based on a validated UA evaluation method, emphasizing the importance of a standardized head- and tongue posture during 3D radiographic image acquisition. An assessment of reliability was not performed in this study since the applied UA evaluation protocol was already validated on CBCT. Nevertheless, implying different scanning techniques may present a limitation although the assessment of reliability based on a large patient sample is the aim of a future study.

We applied a CBCT UA evaluation protocol to validate the anatomical bony landmarks in the MRI images supplemented by the sagittal CBCT scans. Differences between MRI and CBCT images imply variations in scanning time, slice thicknesses and increment. Maintenance of a 
consistent head posture during image acquisition despite the use of head coils and differences in the flexibility and morphology of the cervical vertebrae among the participants were other influencing factors.

\section{Conclusions}

Within the scope of the limitations, the present study demonstrated by applying a standardized and validated UA evaluation method, that altered head or tongue posture during MRI image acquisition had a significant impact on the UA volumes. The head and tongue posture proved to be confounding variables during 3D image acquisition and UA evaluation. These findings are essential for the purposes of 3D UA evaluation and comparison. This study highlights the importance of a standardized head and tongue posture during 3D image acquisition for UA assessment, which may in the context of standardization also be applied to other cephalometric studies.

\section{Declaration of interest: None}

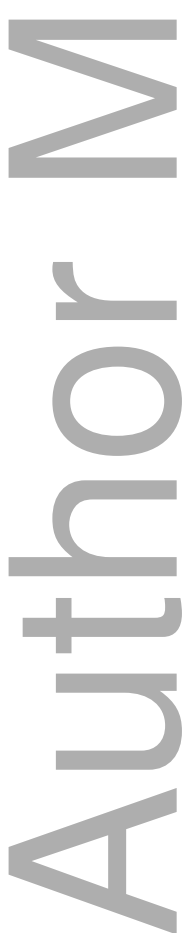


Table I. Descriptive data presenting the obtained retropalatal, oropharyngeal and the total volumes ( $\mathrm{mm}^{3}$ ) from the MRI scans of the included 10 subjects in different head and tongue postures. In NHP, head extension and head flexion, the tongue was maintained in a resting position with the tip of the tongue in contact with the lingual aspect of the lower incisors.

NHP: Supine neutral head position

THP: The tip of the tongue in contact with the posterior edge of the hard palate

TFM: The tip of the tongue in contact with the floor of the mouth in contact with Caruncula sublingualis

\begin{tabular}{|c|c|c|c|c|c|c|}
\hline \multicolumn{2}{|c|}{ Head and tongue position } & \multirow{2}{*}{$\begin{array}{c}\text { NHP } \\
7098\end{array}$} & \multirow{2}{*}{$\begin{array}{c}\text { Head extension } \\
7712\end{array}$} & \multirow{2}{*}{$\begin{array}{c}\text { Head flexion } \\
6291\end{array}$} & \multirow{2}{*}{$\begin{array}{c}\text { NHP with THP } \\
7641\end{array}$} & \multirow{2}{*}{$\begin{array}{c}\text { NHP with TFM } \\
7274\end{array}$} \\
\hline 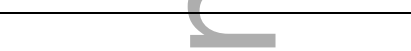 & Mean volume & & & & & \\
\hline & SD & 1660 & 1876 & 1692 & 1949 & 1977 \\
\hline & \multirow{2}{*}{$95 \% \mathrm{CI}$} & 1142 & 1291 & 1164 & 1341 & 1360 \\
\hline & & 3031 & 3426 & 3089 & 3559 & 3609 \\
\hline 2 & Mean volume & 7221 & 8259 & 4585 & 9353 & 7274 \\
\hline \multirow{3}{*}{ Oropharyngeal volume } & SD & 3494 & 4258 & 1664 & 3986 & 2457 \\
\hline & \multirow{2}{*}{$95 \%$ CI } & 2403 & 1291 & 1145 & 1341 & 1360 \\
\hline & & 6379 & 3426 & 3038 & 3559 & 3609 \\
\hline \multirow{4}{*}{ Total volume } & Mean volume & 14388 & 15980 & 10876 & 16995 & 13532 \\
\hline & SD & 4492 & 5591 & 2999 & 4169 & 3893 \\
\hline & \multirow{2}{*}{$95 \%$ CI } & 3090 & 3845 & 2063 & 2868 & 2678 \\
\hline & & 8201 & 10206 & 5474 & 7612 & 7107 \\
\hline
\end{tabular}

This article is protected by copyright. All rights reserved 
Table II. Statistical test results comprising the Wilcoxon Signed-rank test of the UA volume differences between the baseline head- and tongue posture (NHP with TRP) and the other specified head- and tongue postures.

NHP: Supine neutral head position

TRP: Tongue in resting position (with the tip of the tongue in contact with the lingual aspect of the lower incisors)

\section{$\underline{\text { Retropalatal volume }}$}

\begin{tabular}{|c|c|c|c|c|c|c|}
\hline Altered head and tongue posture combinations & $\begin{array}{l}\text { Mean volume } \\
\text { differences } \\
\qquad\left(\mathrm{mm}^{\mathbf{3}}\right)\end{array}$ & $\begin{array}{l}\text { Percentage } \\
\text { change } \\
(\approx \%)\end{array}$ & SD & \multicolumn{2}{|c|}{$95 \% \mathrm{CI}$} & p value \\
\hline $\begin{array}{l}\text { NHP with TRP } \\
\text { Versus } \\
\text { Head extension with the tip of the tongue in contact with } \\
\text { the lingual aspect of the lower incisors }\end{array}$ & 614 & +9 & 353 & 362 & 867 & .005 \\
\hline $\begin{array}{l}\text { NHP with TRP } \\
\text { Head flexion with the tip of the tongue in contact with the } \\
\text { lingual aspect of the lower incisors }\end{array}$ & -807 & -11 & 670 & -1286 & -328 & .006 \\
\hline $\begin{array}{l}\text { NHP with TRP } \\
\text { versus } \\
\text { NHP with the tip of the tongue in contact with the floor } \\
\text { of the mouth in contact with Caruncula sublingualis. }\end{array}$ & 177 & +2 & 740 & -352 & 706 & .38 \\
\hline
\end{tabular}

\section{Oropharyngeal volume}

\begin{tabular}{|l|c|c|c|c|c|}
\hline Altered head and tongue posture combinations & $\begin{array}{c}\text { Mean volume } \\
\text { differences } \\
\left(\mathrm{mm}^{3}\right)\end{array}$ & $\begin{array}{c}\text { Percentage } \\
\text { change } \\
(\approx \%)\end{array}$ & 95\% CI & palue \\
\hline
\end{tabular}




\begin{tabular}{|c|c|c|c|c|c|c|}
\hline $\begin{array}{l}\text { NHP with TRP } \\
\text { Versus } \\
\text { Head extension with the tip of the tongue in contact with } \\
\text { the lingual aspect of the lower incisors }\end{array}$ & 1037 & +14 & 1427 & 17 & 2058 & .03 \\
\hline $\begin{array}{l}\text { NHP with TRP } \\
\text { versus } \\
\text { Head flexion with the tip of the tongue in contact with the } \\
\text { lingual aspect of the lower incisors }\end{array}$ & -2636 & -36 & 2250 & -4245 & -1027 & .009 \\
\hline $\begin{array}{l}\text { NHP with TRP } \\
\text { versus } \\
\text { NHP with the tip of the tongue in contact with the } \\
\text { posterior edge of the hard palate }\end{array}$ & 2132 & +30 & 3818 & -599 & 4863 & .05 \\
\hline
\end{tabular}

\section{$\underline{\text { Total volume }}$}

\begin{tabular}{|c|c|c|c|c|c|c|}
\hline Altered head and tongue posture combinations & $\begin{array}{l}\text { Mean volume } \\
\text { differences } \\
\qquad\left(\mathrm{mm}^{3}\right)\end{array}$ & $\begin{array}{l}\text { Percentage } \\
\text { change } \\
(\approx \%)\end{array}$ & SD & \multicolumn{2}{|c|}{$95 \% \mathrm{CI}$} & p value \\
\hline $\begin{array}{l}\text { NHP with TRP } \\
\text { Versus } \\
\text { Head extension with the tip of the tongue in contact with } \\
\text { the lingual aspect of the lower incisors }\end{array}$ & 1592 & +11 & 1657 & 407 & 2777 & .02 \\
\hline $\begin{array}{l}\text { NHP with TRP } \\
\text { Head flexion with the tip of the tongue in contact with the } \\
\text { lingual aspect of the lower incisors }\end{array}$ & -3511 & -24 & 2445 & -5260 & -1762 & .005 \\
\hline $\begin{array}{l}\text { NHP with TRP } \\
\text { versus } \\
\text { NHP with the tip of the tongue in contact with the floor } \\
\text { of the mouth in contact with Caruncula sublingualis. }\end{array}$ & -856 & -5 & 2096 & -2355 & 643 & .33 \\
\hline
\end{tabular}




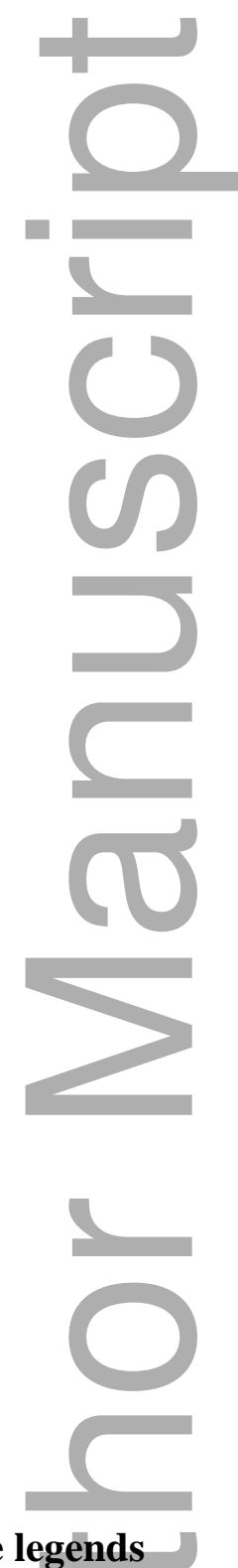

\section{Figure legends}

Figure 1. MRI and supine CBCT scan from same subject, presenting the image acquisition protocol for altered head and tongue postures and the obtained UA volume compartments. In NHP, head extension and head flexion, the tongue was maintained in a resting position with the tip of the tongue in contact with the lingual aspect of the lower incisors

NHP: Supine neutral head position

THP: The tip of the tongue in contact with the posterior edge of the hard palate

TFM: The tip of the tongue in contact with the floor of the mouth in contact with Caruncula sublingualis 
Figure 2. MRI scans from same subject in supine neutral head position, representing the upper airway protocol. (A) shows some of the anatomical landmarks used to define the reference planes (B) in order to delineate and subdivide the upper airway compartments (C).

(A) Anatomical landmarks

OrR: orbitale right, OrL: orbitale left, PoR: porion right, PoL: porion left, S: sella turcica,

$\mathbf{N}$ : nasion, Ba: basion, So: midpoint of the sella-basion line and $\mathbf{E}$ : epiglottis

(B) Reference planes

Frankfurt horizontal plane

Plane passing through the inferior borders of the bony orbits, encompassed by OrR and OrL, and the upper margin of the auditory meatus encompassed by PoR and PoL.

\section{Sagittal SN plane}

Plane perpendicular to the Frankfurt horizontal plane and passing through $\mathrm{S}$ and $\mathrm{N}$ points

\section{SN Horizontal plane}

Plane passing through the S- and N points and perpendicular to the Sagittal SN plane

\section{SBa Coronal plane}

Plane passing through the S- and Ba points and perpendicular to the Sagittal SN plane

\section{Airway superior border 1 plane}

Plane passing through So and perpendicular to the S-Ba Coronal- and Sagittal SN planes

\section{Airway superior border 2 plane}

Plane passing through $\mathrm{Ba}$ and parallel to the Airway superior border 1 plane

\section{Airway inferior border plane}

Plane passing through E and parallel to the Frankfurt horizontal plane

(C) Upper airway compartments

Blue compartment represents the retropalatal volume and red compartment represents the oropharyngeal volume, which added comprise the total volume.

\section{References}

1. Di Carlo G, Polimeni A, Melsen B, Cattaneo PM. The relationship between upper airways and craniofacial morphology studied in 3D. A CBCT study. Orthod Craniofac Res [Internet]. 2015 Feb;18(1):1-11.

2. Guijarro-Martínez R, Swennen GRJ. Cone-beam computerized tomography imaging and analysis of the upper airway: A systematic review of the literature. Int J Oral Maxillofac Surg. 2011;40(11):1227-37. 
3. De Felice F, Di Carlo G, Saccucci M, Tombolini V, Polimeni A. Dental Cone Beam Computed Tomography in Children: Clinical Effectiveness and Cancer Risk due to Radiation Exposure. Oncology [Internet]. 2019 Mar 5;96(4):1-6.

4. Brenner DJ, Hall EJ. Computed tomography--an increasing source of radiation exposure. N Engl J Med [Internet]. 2007 Nov 29;357(22):2277-84.

5. Schwab RJ. Upper airway imaging. Clin Chest Med [Internet]. 1998 Mar;19(1):33-54.

6. Shin LK, Holbrook AB, Capasso R, Kushida CA, Powell NB, Fischbein NJ, et al. Improved sleep MRI at 3 tesla in patients with obstructive sleep apnea. J Magn Reson Imaging. 2013;38(5):1261-6.

7. Schwab RJ, Gupta KB, Gefter WB, Metzger LJ, Hoffman EA, Pack AI. Upper airway and soft tissue anatomy in normal subjects and patients with sleep-disordered breathing. Significance of the lateral pharyngeal walls. Am J Respir Crit Care Med [Internet]. 1995 Nov;152(5 Pt 1):1673-89.

8. Schwab RJ, Gefter WB, Hoffman EA, Gupta KB, Pack AI. Dynamic upper airway imaging during awake respiration in normal subjects and patients with sleep disordered breathing. Am Rev Respir Dis [Internet]. 1993 Nov;148(5):1385-400.

9. Ayappa I, Rapoport DM. The upper airway in sleep: Physiology of the pharynx. Sleep Medicine Reviews. 2003. p. 9-33.

10. Otsuka R, Ono T, Ishiwata Y, Kuroda T. Respiratory-related genioglossus electromyographic activity in response to head rotation and changes in body position. Angle Orthod [Internet]. 2000 Feb;70(1):63-9.

11. Ono T, Otsuka R, Kuroda T, Honda E, Sasaki T. Effects of head and body position on two- and three-dimensional configurations of the upper airway. J Dent Res [Internet]. 2000 Nov;79(11):1879-84.

12. Moorrees, C. F. A. and Kean MR. Natural head position, a basic consideration in the interpretation of cephalometric radiographs. Am J Phys Anthropol,. 1958;16: 213-234.

13. Verma SK, Maheshwari S, Gautam SN, Prabhat K, Kumar S. Natural head position: key position for radiographic and photographic analysis and research of craniofacial complex. J Oral Biol Craniofacial Res [Internet]. 2012 Jan;2(1):46-9.

14. Solow B, Sandham A. Cranio-cervical posture: a factor in the development and function of the dentofacial structures. Eur J Orthod [Internet]. 2002 Oct;24(5):447-56.

15. Hellsing E. Changes in the pharyngeal airway in relation to extension of the head. Eur J Orthod [Internet]. 1989 Nov;11(4):359-65.

16. Muto T, Takeda S, Kanazawa M, Yamazaki A, Fujiwara Y, Mizoguchi I. The effect of 
head posture on the pharyngeal airway space (PAS). Int J Oral Maxillofac Surg [Internet]. 2002 Dec;31(6):579-83.

17. Cattaneo PM, Melsen B. The use of cone-beam computed tomography in an orthodontic department in between research and daily clinic. World J Orthod [Internet]. 2008;9(3):269-82.

18. Lenza MG, Lenza MMDO, Dalstra M, Melsen B, Cattaneo PM. An analysis of different approaches to the assessment of upper airway morphology: a CBCT study. Orthod Craniofac Res [Internet]. 2010 May;13(2):96-105.

19. Aboudara C, Nielsen I, Huang JC, Maki K, Miller AJ, Hatcher D. Comparison of airway space with conventional lateral headfilms and 3-dimensional reconstruction from cone-beam computed tomography. Am J Orthod Dentofacial Orthop [Internet]. 2009 Apr; 135(4):468-79.

20. Gurani SF, Di Carlo G, Cattaneo PM, Thorn JJ, Pinhol EM. Effect of Head and Tongue Posture on the Pharyngeal Airway Dimensions and Morphology in ThreeDimensional Imaging: a Systematic Review. J Oral Maxillofac Res [Internet]. 2016 Mar 31;7(1):e1.

21. Pirilä-Parkkinen K, Pirttiniemi P, Pääkkö E, Tolonen U, Nieminen P, Löppönen H. Pharyngeal airway in children with sleep-disordered breathing in relation to head posture. Sleep Breath [Internet]. 2012 Sep;16(3):737-46.

22. Zhang W, Song X, Masumi SI, Tanaka T, Zhu Q. Effects of head and body positions on 2-and 3-dimensional configuration of the oropharynx with jaw protruded: a magnetic resonance imaging study. Oral Surg Oral Med Oral Pathol Oral Radiol Endod [Internet]. Elsevier Inc.; 2011 Jun;111(6):778-84.

23. Gokce SM, Gorgulu S, Gokce HS, Bengi AO, Karacayli U, Ors F. Evaluation of pharyngeal airway space changes after bimaxillary orthognathic surgery with a 3dimensional simulation and modeling program. Am J Orthod Dentofac Orthop. American Association of Orthodontists; 2014;146(4):477-92.

24. Alsufyani NA, Al-Saleh MAQ, Major PW. CBCT assessment of upper airway changes and treatment outcomes of obstructive sleep apnoea: a systematic review. Sleep Breath [Internet]. 2013 Sep;17(3):911-23.

25. Alsufyani NA, Dietrich NH, Lagravère MO, Carey JP, Major PW. Cone beam computed tomography registration for 3-D airway analysis based on anatomic landmarks. Oral Surg Oral Med Oral Pathol Oral Radiol [Internet]. 2014 Sep;118(3):371-83. 
26. Cai M, Brown EC, Hatt A, Cheng S, Bilston LE. Effect of head and jaw position on respiratory-related motion of the genioglossus. J Appl Physiol [Internet]. 2016 Apr;120(7):758-65.

27. Kobayashi I, Perry A, Rhymer J, Wuyam B, Hughes P, Murphy K, et al. Inspiratory coactivation of the genioglossus enlarges retroglossal space in laryngectomized humans. J Appl Physiol [Internet]. 1996;80(5):1595-604.

28. Edwards BA, White DP. Control of the pharyngeal musculature during wakefulness and sleep: Implications in normal controls and sleep apnea. Head Neck [Internet]. 2011 Oct;33(S1):S37-45.

29. Ono T. Tongue and upper airway function in subjects with and without obstructive sleep apnea. Jpn Dent Sci Rev [Internet]. 2012 Aug;48(2):71-80.

30. Sutthiprapaporn P, Tanimoto K, Ohtsuka M, Nagasaki T, Iida Y, Katsumata a. Positional changes of oropharyngeal structures due to gravity in the upright and supine positions. Dentomaxillofacial Radiol. 2008;37(3):130-6.

31. Di Carlo G, Gurani SF, Pinholt EM, Cattaneo PM. A new simple three-dimensional method to characterize upper airway in orthognathic surgery patient. Dentomaxillofac Radiol [Internet]. 2017 Dec;46(8):20170042.

32. Gurani SF, Di Carlo G, Thorn JJ, Ingerslev J, Cattaneo PM, Pinholt EM. Two-Year Postoperative Upper Airway Cone-Beam Computed Tomographic Outcomes Based on a Verified Upper Airway Analysis Following Bimaxillary Orthognathic Surgery. J Oral Maxillofac Surg [Internet]. W.B. Saunders; 2019 Jul 1;77(7):1435-45.

33. Alsufyani NA, Noga ML, Witmans M, Cheng I, El-Hakim H, Major PW. Using cone beam CT to assess the upper airway after surgery in children with sleep disordered breathing symptoms and maxillary-mandibular disproportions: a clinical pilot. J Otolaryngol - Head Neck Surg [Internet]. 2017 Dec 11;46(1):31.

34. Alsufyani NA, Noga ML, Witmans M, Major PW. Upper airway imaging in sleepdisordered breathing: role of cone-beam computed tomography. Oral Radiol [Internet]. 2017 Sep 20;33(3):161-9.

35. Guijarro-Martínez R, Swennen GRJ. Three-dimensional cone beam computed tomography definition of the anatomical subregions of the upper airway: a validation study. Int J Oral Maxillofac Surg [Internet]. 2013 Sep;42(9):1140-9.

36. Sears CR, Miller AJ, Chang MK, Huang JC, Lee JS. Comparison of pharyngeal airway changes on plain radiography and cone-beam computed tomography after orthognathic surgery. J Oral Maxillofac Surg. Elsevier Inc.; 2011;69(11):e385-94. 
37. Hernández-Alfaro F, Guijarro-Martínez R, Mareque-Bueno J. Effect of mono- and bimaxillary advancement on pharyngeal airway volume: Cone-beam computed tomography evaluation. J Oral Maxillofac Surg. 2011;69(11):e395-400.

38. Abramson Z, Susarla SM, Lawler M, Bouchard C, Troulis M, Kaban LB. Threedimensional computed tomographic airway analysis of patients with obstructive sleep apnea treated by maxillomandibular advancement. J Oral Maxillofac Surg [Internet]. Elsevier Inc.; 2011;69(3):677-86.

39. Shin JH, Kim MA, Park IY, Park YH. A 2-year follow-up of changes after bimaxillary surgery in patients with mandibular prognathism: 3-dimensional analysis of pharyngeal airway volume and hyoid bone position. J Oral Maxillofac Surg [Internet]. American Association of Oral and Maxillofacial Surgeons; 2015;73(2):340.e1-340.e9.

40. Rubio-Bueno P, Landete P, Ardanza B, Vázquez L, Soriano JB, Wix R, et al. Maxillomandibular advancement as the initial treatment of obstructive sleep apnoea: Is the mandibular occlusal plane the key? Int J Oral Maxillofac Surg [Internet]. 2017 Nov;46(11):1363-71.

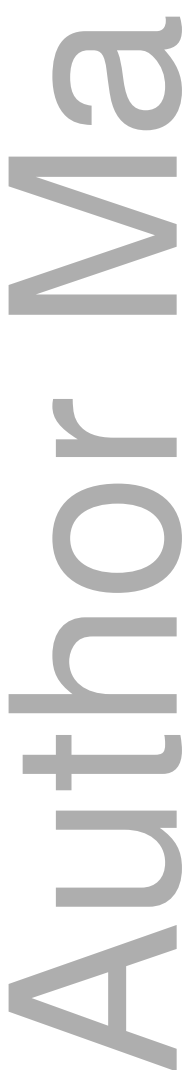


Natural Head positico

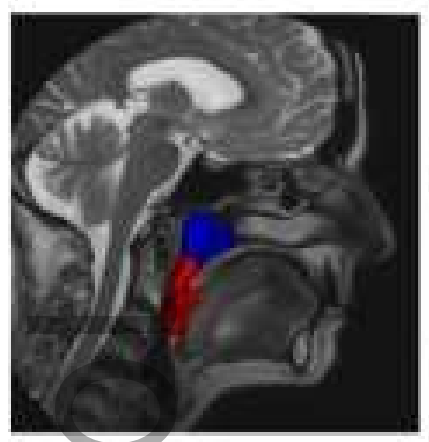

r
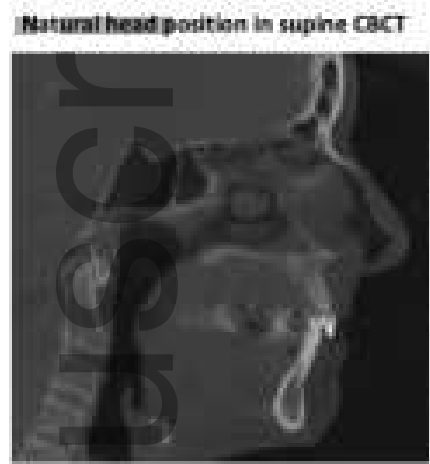

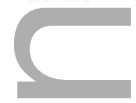
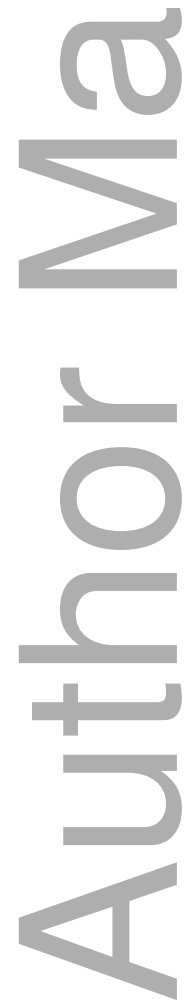

Head extansien

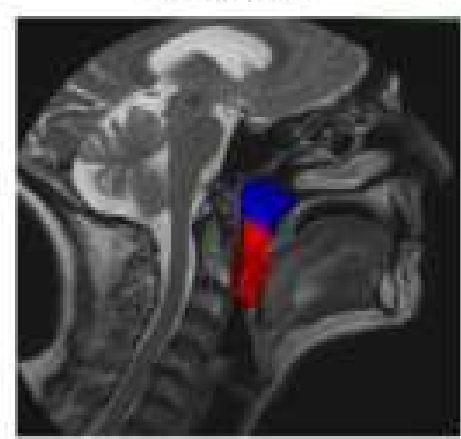

NRP with THP

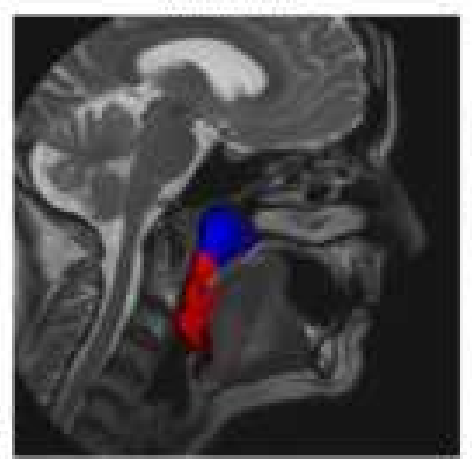

ocr_12348_f1.tiff

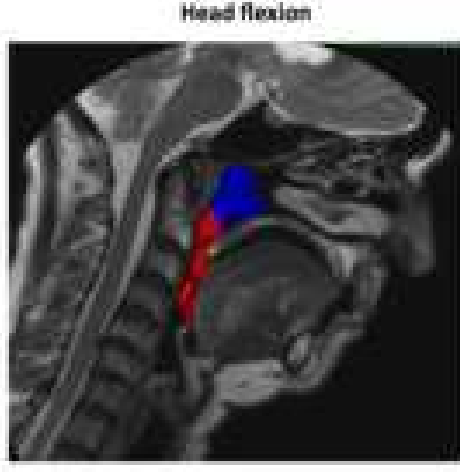

NHP with THM

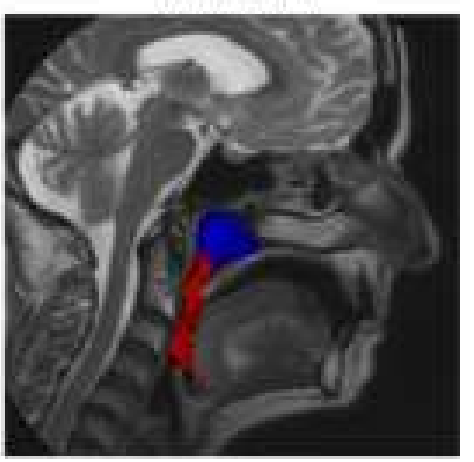




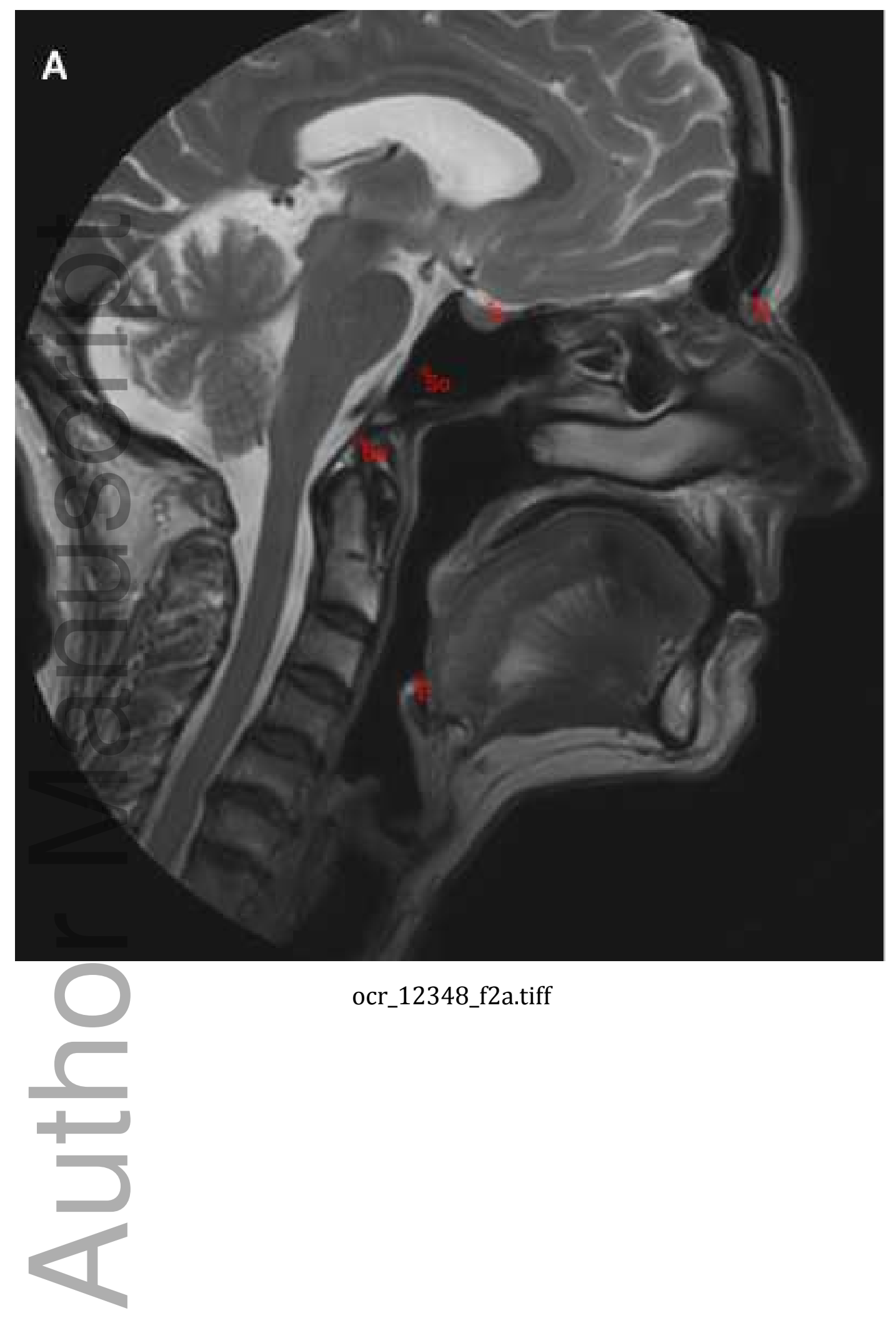

This article is protected by copyright. All rights reserved 


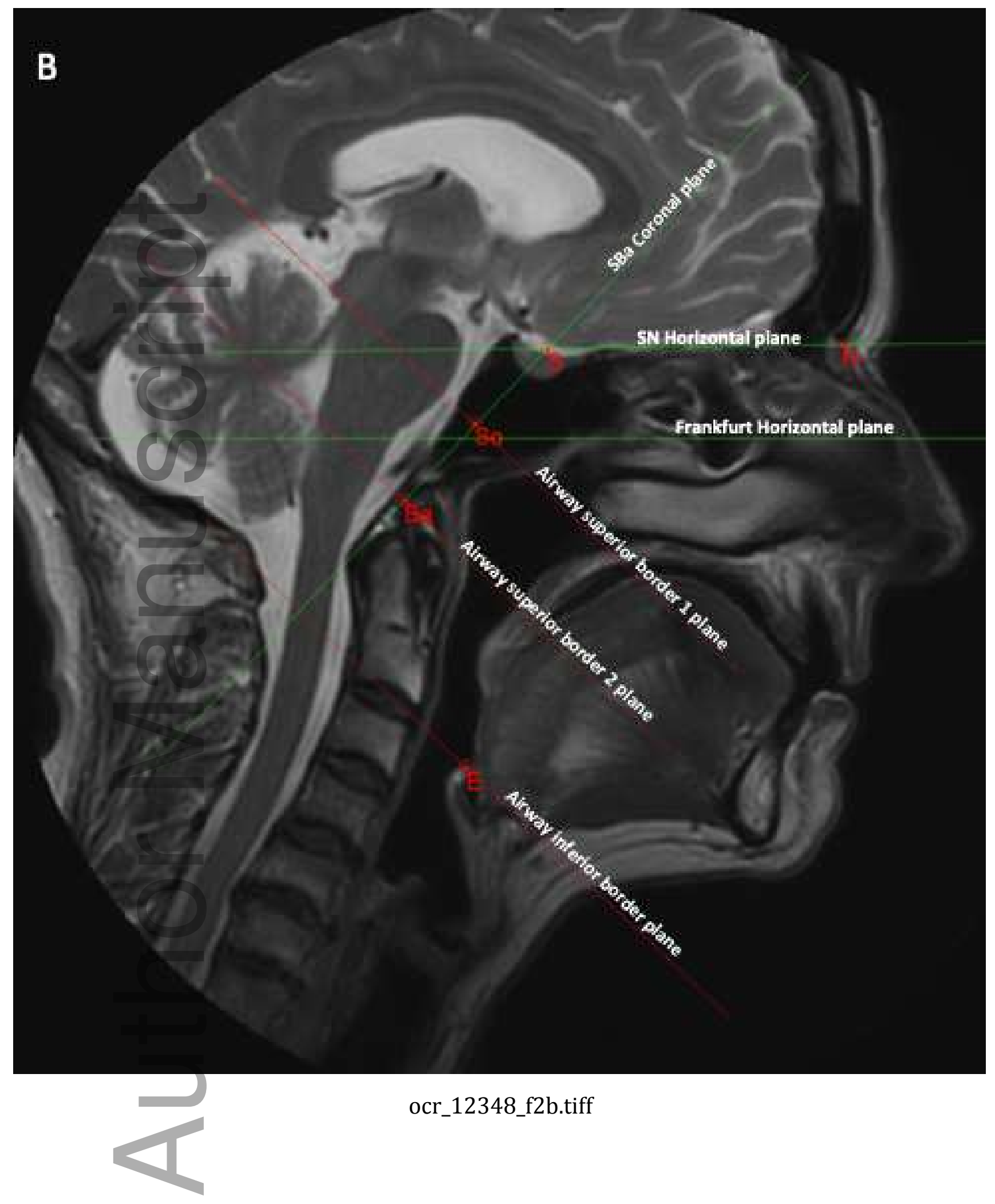

This article is protected by copyright. All rights reserved 


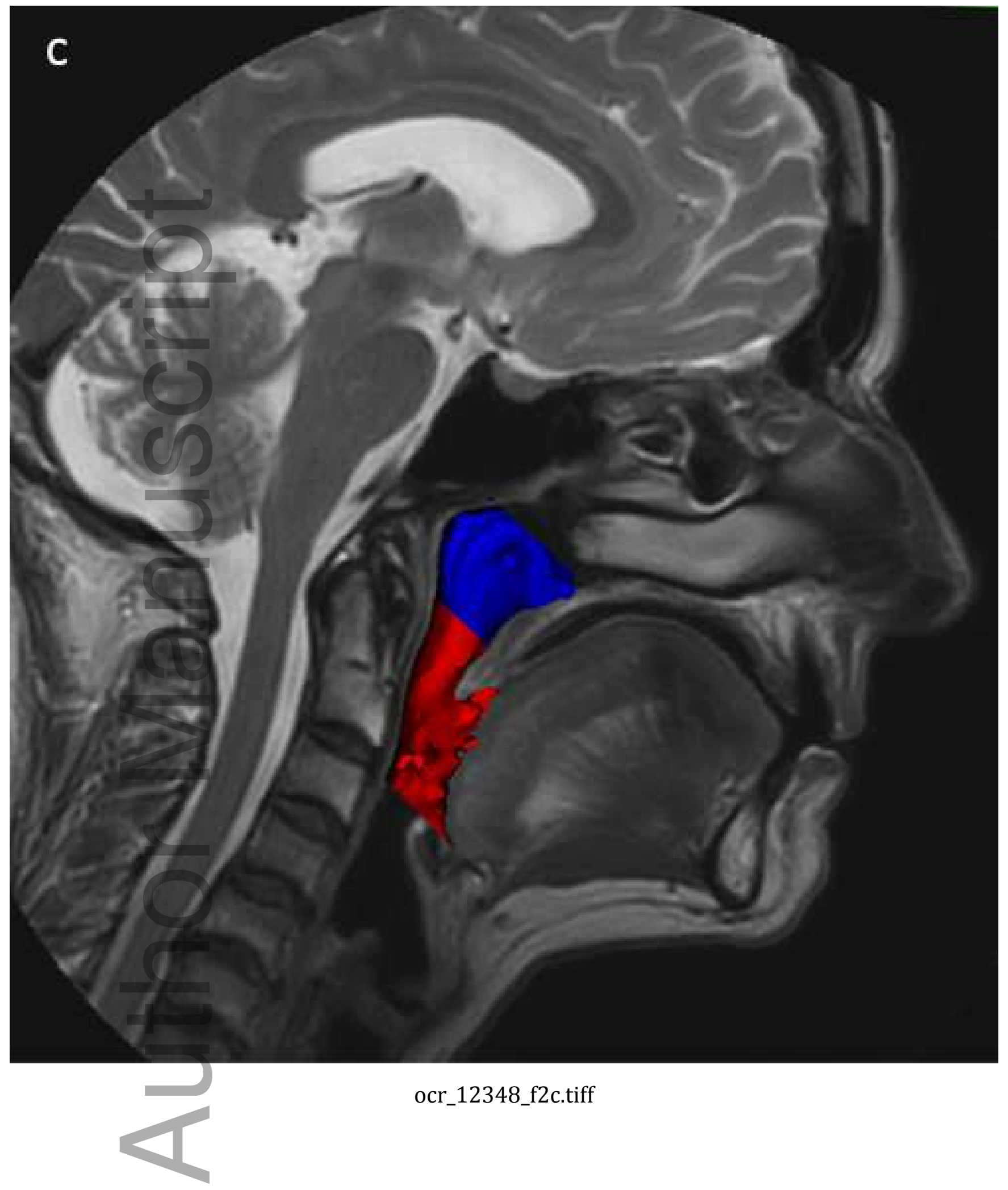

This article is protected by copyright. All rights reserved 


\section{University Library}

\section{- M M N E R VA A gateway to Melbourne's research publications}

Minerva Access is the Institutional Repository of The University of Melbourne

Author/s:

Gurani, SF;Cattaneo, PM;Rafaelsen, SR;Pedersen, MR;Thorn, JJ;Pinholt, EM

Title:

The effect of altered head and tongue posture on upper airway volume based on a validated upper airway analysis-An MRI pilot study

Date:

2019-11-20

\section{Citation:}

Gurani, S. F., Cattaneo, P. M., Rafaelsen, S. R., Pedersen, M. R., Thorn, J. J. \& Pinholt, E. M. (2019). The effect of altered head and tongue posture on upper airway volume based on a validated upper airway analysis-An MRI pilot study. ORTHODONTICS \& CRANIOFACIAL RESEARCH, 23 (1), pp.102-109. https://doi.org/10.1111/ocr.12348.

Persistent Link:

http://hdl.handle.net/11343/286641 